\title{
Supersymmetric Extension of Non-Hermitian su(2) Hamiltonian and Supercoherent States ${ }^{\star}$
}

\author{
Omar CHERBAL ${ }^{\dagger}$, Mahrez DRIR ${ }^{\dagger}$, Mustapha MAAMACHE ${ }^{\ddagger}$ and Dimitar A. TRIFONOV $\S$ \\ $\dagger$ Faculty of Physics, Theoretical Physics Laboratory, USTHB, \\ B.P. 32, El Alia, Algiers 16111, Algeria \\ E-mail: ocherbal@yahoo.fr,ocherbal@usthb.dz \\ $¥$ Laboratoire de Physique Quantique et Systemes Dynamiques, Department of Physics, \\ Setif University, Setif 19000, Algeria \\ E-mail: maamache_m@yahoo.fr \\ $\S$ Institute of Nuclear Research, 72 Tzarigradsko chaussée, 1784 Sofia, Bulgaria \\ E-mail: dtrif@inrne.bas.bg
}

Received September 29, 2010, in final form December 04, 2010; Published online December 15, 2010 doi:10.3842/SIGMA.2010.096

\begin{abstract}
A new class of non-Hermitian Hamiltonians with real spectrum, which are written as a real linear combination of $\mathrm{su}(2)$ generators in the form $H=\omega J_{3}+\alpha J_{-}+\beta J_{+}$, $\alpha \neq \beta$, is analyzed. The metrics which allows the transition to the equivalent Hermitian Hamiltonian is established. A pseudo-Hermitian supersymmetic extension of such Hamiltonians is performed. They correspond to the pseudo-Hermitian supersymmetric systems of the boson-phermion oscillators. We extend the supercoherent states formalism to such supersymmetic systems via the pseudo-unitary supersymmetric displacement operator method. The constructed family of these supercoherent states consists of two dual subfamilies that form a bi-overcomplete and bi-normal system in the boson-phermion Fock space. The states of each subfamily are eigenvectors of the boson annihilation operator and of one of the two phermion lowering operators.
\end{abstract}

Key words: pseudo-Hermitian quantum mechanics; supersymmetry; supercoherent states

2010 Mathematics Subject Classification: 81Q12; 81Q60; 81R30

\section{Introduction}

The study of non-Hermitian Hamiltonians with real spectrum has received a great deal of interest during the last decade $[1,2]$. One celebrated model of such non-Hermitian PT-symmetric Hamiltonian is proposed by Swanson [3], which is expressed in terms of the usual harmonic oscillator creation and annihilation operators $a^{\dagger}$ and $a$, namely $H=\omega\left(a^{\dagger} a+\frac{1}{2}\right)+\alpha a^{2}+\beta a^{\dagger 2}$ with $\omega, \alpha$, and $\beta$ real parameters, such that $\alpha \neq \beta$ and $\omega^{2}-4 \alpha \beta>0$. This Hamiltonian has been studied extensively in the literature by several authors $[3,4,5,6,7]$. The metric operator $\rho$, mapping $H$ to its Hermitian counterpart $h$ via the relation $h=\rho H \rho^{-1}$, has been constructed by using several approaches. This Hamiltonian has been extended later on by Quesne [8, 9] in the framework of the $\mathrm{su}(1,1)$ approach by writing it as a linear combination of $\mathrm{su}(1,1)$ generators.

In the context of this extension, we introduce another kind of non-Hermitian Hamiltonian with real spectrum in the Lie-algebric framework $[10,11]$, which is presented as a linear combination of the generators $J_{-}, J_{+}$and $J_{3}$ of the su(2) Lie algebra. Then we introduce the pseudo-Hermitian

${ }^{\star}$ This paper is a contribution to the Proceedings of the Workshop "Supersymmetric Quantum Mechanics and Spectral Design" (July 18-30, 2010, Benasque, Spain). The full collection is available at http://www.emis.de/journals/SIGMA/SUSYQM2010.html 
supersymmetric extension of such Hamiltonians, and naturally extend the supercoherent states approach to such pseudo-Hermitian supersymmetric systems. The Hermitian version of such Hamiltonians has been widely used in the fields of atomic physics and quantum optics, in particular in the study of the interaction of two-level atom systems with a coherent radiation field $[12,13,14,15]$.

The organization of the paper is as follows. In Section 2 we study our pseudo-Hermitian Hamiltonian and we establish the metrics which allows the transition to the corresponding Hermitian one. In Section 3 we consider a pseudo-Hermitian supersymmetric system in the form of boson-phermion ${ }^{1}$ oscillator. In Section 4 we construct the supercoherent states (SCS) from the lowest (ground) eigenstates of the supersymmetric Hamiltonians $H_{s}$ and $H_{s}^{\dagger}$, by acting with a pair of the pseudo-unitary displacement operators. We show that these SCS are eigenstates of the boson annihilation operator and of the pair of phermion annihilation operators. The set of such SCS form a bi-normalized and bi-overcomplete system. The paper ends with concluding remarks.

\section{Non-Hermitian su(2) Hamiltonian}

We consider the following non-Hermitian Hamiltonian:

$$
H=\omega\left(Y^{\dagger} Y-\frac{1}{2}\right)+\alpha Y+\beta Y^{\dagger}
$$

where $\omega, \alpha$, and $\beta$ are real parameters such that $\alpha \neq \beta$ and $\omega^{2}+4 \alpha \beta>0, Y$ and $Y^{\dagger}$ are fermion annihilation and creation operators respectively, which obey the usual fermion algebra:

$$
\left\{Y, Y^{\dagger}\right\} \equiv Y Y^{\dagger}+Y^{\dagger} Y=1, \quad Y^{2}=Y^{\dagger 2}=0 .
$$

The Hamiltonian (2.1) is the non-Hermitian extension of the Hermitian fermionic Hamiltonian studied in greater detail in our recent work [17]. Due to the nilpotency of the fermionic operators $Y$ and $Y^{\dagger}$, the Hamiltonian (2.1) which is a linear combination of $Y, Y^{\dagger}$ and $\left(Y^{\dagger} Y-\frac{1}{2}\right)$, represents the most general form of such non-Hermitian Hamiltonians. The three operators $Y, Y^{\dagger}$ and $\left(Y^{\dagger} Y-\frac{1}{2}\right)$ close under commutation the su(2) Lie algebra:

$$
\left[J_{+}, J_{-}\right]=2 J_{3}, \quad\left[J_{3}, J_{ \pm}\right]= \pm J_{ \pm}
$$

where

$$
J_{+}=Y^{\dagger}, \quad J_{-}=Y, \quad J_{3}=Y^{\dagger} Y-\frac{1}{2},
$$

and $J_{3}^{\dagger}=J_{3}, J_{ \pm}^{\dagger}=J_{\mp}$. Thus the Hamiltonian (2.1) is expressed as:

$$
H=\omega J_{3}+\alpha J_{-}+\beta J_{+} .
$$

It would be useful to mention that the Hermitian version of the $\mathrm{su}(2)$ Hamiltonian (2.2), has been widely used in the fields of atomic and optical physics, and quantum optics, in the study of systems of two-level atoms interacting resonantly with a coherent radiation field $[12,13,14,15]$. For this reason in the present paper we are interesting in investigation of the supercoherent state formalism (in Section 4).

\footnotetext{
${ }^{1}$ The notion of pseudo-fermion (phermion) has been introduced by Mostafazadeh [16]. The defining algebra of the phermions is a pseudo-Hermitian generalization of the usual fermion algebra, namely: $\alpha^{2}=\alpha^{\# 2}=0$, $\alpha \alpha^{\#}+\alpha^{\#} \alpha=1$, where $\alpha^{\#}=\eta^{-1} \alpha^{\dagger} \eta$ and $\alpha$ are respectively the creation and annihilation operators of what was called the pseudo-Hermitian fermion or simply a phermion [16], and $\eta$ is a linear, Hermitian, and invertible operator. At $\eta=1$ one has $\alpha^{\#}=\alpha^{\dagger}$, i.e. the phermion algebra reduces to the usual fermion algebra.
} 
Following the procedure as in $[8,9]$, the non-Hermitian operator $H$ can be transformed into the corresponding Hermitian Hamiltonian $h$ by means of the similarity transformation

$$
h=\rho H \rho^{-1} .
$$

This means that $H$ admits positive-definite metric operator $\eta_{+}=\rho^{2}$. We look for the mapping function $\rho$ in the form

$$
\rho=e^{\epsilon\left[2 J_{3}+z\left(J_{-}+J_{+}\right)\right]}
$$

where $\epsilon$ and $z$ are real parameters. By using $2 \times 2$ matrix representation of $J_{+}, J_{-}$, and $J_{3}$, namely

$$
J_{+}=\left(\begin{array}{cc}
0 & 1 \\
0 & 0
\end{array}\right), \quad J_{-}=\left(\begin{array}{cc}
0 & 0 \\
1 & 0
\end{array}\right), \quad J_{3}=\left(\begin{array}{cc}
\frac{1}{2} & 0 \\
0 & -\frac{1}{2}
\end{array}\right),
$$

we find

$$
\rho=e^{\epsilon\left[2 J_{3}+z\left(J_{-}+J_{+}\right)\right]}=\left(\begin{array}{cc}
\cosh \theta+\epsilon(\sinh \theta) / \theta & \epsilon z(\sinh \theta) / \theta \\
\epsilon z(\sinh \theta) / \theta & \cosh \theta-\epsilon(\sinh \theta) / \theta
\end{array}\right),
$$

where $\theta=\epsilon \sqrt{1+z^{2}}$, and $\epsilon$ and $z$ are related through formula

$$
\epsilon=\frac{1}{2 \sqrt{1+z^{2}}} \operatorname{arctanh} \frac{(\alpha-\beta) \sqrt{1+z^{2}}}{\alpha+\beta-\omega z}, \quad z \in \mathbb{R} .
$$

The mapping $\rho$ can also be written in the form

$$
\rho=\left(\frac{\alpha+\beta-\omega z+(\alpha-\beta) \sqrt{1+z^{2}}}{\alpha+\beta-\omega z-(\alpha-\beta) \sqrt{1+z^{2}}}\right)^{\frac{1}{4 \sqrt{1+z^{2}}}\left[2 J_{3}+z\left(J_{-}+J_{+}\right)\right]} .
$$

Introducing (2.5) into (2.3) we obtain the Hermitian $h$ in the form,

$$
h=\delta J_{3}+\lambda\left(J_{-}+J_{+}\right)
$$

where $\delta$ and $\lambda$ are given explicitly by

$$
\begin{aligned}
& \delta=\frac{\omega+(\alpha+\beta) z-z(\alpha+\beta-\omega z) \sqrt{1-\frac{(\alpha-\beta)^{2}\left(1+z^{2}\right)}{(\alpha+\beta-\omega z)^{2}}}}{1+z^{2}}, \\
& \lambda=\frac{\omega z+(\alpha+\beta) z^{2}+(\alpha+\beta-\omega z) \sqrt{1-\frac{(\alpha-\beta)^{2}\left(1+z^{2}\right)}{(\alpha+\beta-\omega z)^{2}}}}{2\left(1+z^{2}\right)} .
\end{aligned}
$$

It is worth noting that in terms of parameters $\epsilon, z$ and group generators $J_{i}$ the above formulas are quite similar to the corresponding ones for the case of $\operatorname{su}(1,1)[8,9]$. We would like however to emphasize that our $\theta=\epsilon \sqrt{1+z^{2}}$ is manifestly real and positive, which means that in the $\mathrm{su}(2)$ approach, the positivity of the Hermitian operator $\rho$ is ensured for any $z \in \mathbb{R}$, unlike the $\mathrm{su}(1,1)$ approach case [8,9], where $z$ is restricted to the interval $[-1,1]$. This is the principal difference between the metrics of the two approaches.

We note that formulas (2.4), (2.6) for $\rho$ and (2.2), (2.3), (2.7) for $H$ and $h$ are valid in any Hermitian representation of $J_{i}$. In the case of half integer spin we can further express $h$ in terms of fermionic number operator, and $H$ - in terms of pseudo-Hermitian fermionic (phermionic) 
number operator. In this aim we introduce the creation and annihilation operators $b^{\dagger}$ and $b$ associated to the corresponding Hermitian Hamiltonian $h$ given in equation (2.7) as,

$$
b=\frac{(\delta+\Omega)}{2 \Omega} J_{-}+\frac{(\delta-\Omega)}{2 \Omega} J_{+}-\frac{2 \lambda}{\Omega} J_{3}, \quad b^{\dagger}=\frac{(\delta-\Omega)}{2 \Omega} J_{-}+\frac{(\delta+\Omega)}{2 \Omega} J_{+}-\frac{2 \lambda}{\Omega} J_{3},
$$

where

$$
\Omega=\sqrt{\omega^{2}+4 \alpha \beta} .
$$

The operators $b^{\dagger}$ and $b$ satisfies the standard fermion algebra:

$$
\left\{b, b^{\dagger}\right\} \equiv b b^{\dagger}+b^{\dagger} b=1, \quad b^{2}=b^{\dagger 2}=0 .
$$

In terms of $b$ and $b^{\dagger}$, the Hamiltonian $h$ is factorized to the form of the fermionic oscillator,

$$
h=\Omega\left(b^{\dagger} b-\frac{1}{2}\right),
$$

The number operators $N=b^{\dagger} b$ satisfies

$$
[b, N]=b, \quad\left[b^{\dagger}, N\right]=-b^{\dagger}, \quad\left[b, b^{\dagger}\right]=1-2 N,
$$

The Hilbert space of the single-fermion system is spanned by the two eigenstates $\{|0\rangle,|1\rangle\}$ of $N$ :

$$
b^{\dagger} b|n\rangle=n|n\rangle, \quad n=0,1 .
$$

The operators $b$ and $b^{\dagger}$ allow transitions between the states as

$$
b|0\rangle=0, \quad b|1\rangle=|0\rangle, \quad b^{\dagger}|1\rangle=0, \quad b^{\dagger}|0\rangle=|1\rangle .
$$

Now we can apply to $b$ and $b^{\dagger}$ a similarity transformation, that is inverse to (2.3) to get annihilation and creation operators $B$ and $B^{\#}$ associated to the quasi-Hermitian Hamiltonian (2.2),

$$
B=\rho^{-1} b \rho, \quad B^{\#}=\rho^{-1} b^{\dagger} \rho .
$$

The operators $B$ and $B^{\#}$ are related via the pseudo-Hermitian conjugation, $B^{\#}=\rho^{-2} B^{\dagger} \rho^{2}$ and satisfies the phermion algebra [16]

$$
B^{2}=B^{\# 2}=0, \quad\left\{B, B^{\#}\right\}=B B^{\#}+B^{\#} B=1 .
$$

Using then the equations (2.3), (2.9) and (2.10) we get the pseudo-Hermitian Hamiltonian (2.1) in a factorized form

$$
H=\Omega\left(B^{\#} B-\frac{1}{2}\right) .
$$

The phermionic ladder operators $B$ and $B^{\#}$ can be represented in the form of (non-Hermitian) linear combination of the generators $J_{3}, J_{ \pm}$,

$$
B=\mu_{1} J_{-}+\mu_{2} J_{+}+2 \mu_{3} J_{3}, \quad B^{\#}=\nu_{1} J_{-}+\nu_{2} J_{+}+2 \nu_{3} J_{3},
$$

where $\mu_{1}, \mu_{2}, \mu_{3}, \nu_{1}, \nu_{2}$ and $\nu_{3}$ are expressed in terms of the $H$ - and $\rho$-parameters $\omega, \alpha, \beta$ and $\epsilon, z$ as follows

$$
\mu_{1}=\frac{\delta+\Omega}{2 \Omega}+\left[\left(1+\tau+z^{2}\right) \epsilon \frac{\sinh \theta}{\theta}+(1+\tau) \cosh \theta\right] \epsilon \frac{\sinh \theta}{\theta},
$$




$$
\begin{aligned}
& \mu_{2}=\frac{\delta-\Omega}{2 \Omega}-\left[\left(1-\tau+z^{2}\right) \epsilon \frac{\sinh \theta}{\theta}-(1-\tau) \cosh \theta\right] \epsilon \frac{\sinh \theta}{\theta} \\
& \mu_{3}=-\frac{\lambda}{\Omega}-\left[\tau \epsilon \frac{\sinh \theta}{\theta}+\cosh \theta\right] z \epsilon \frac{\sinh \theta}{\theta} \\
& \nu_{1}=\frac{\delta-\Omega}{2 \Omega}-\left[\left(1-\tau+z^{2}\right) \epsilon \frac{\sinh \theta}{\theta}+(1-\tau) \cosh \theta\right] \epsilon \frac{\sinh \theta}{\theta} \\
& \nu_{2}=\frac{\delta+\Omega}{2 \Omega}+\left[\left(1+\tau+z^{2}\right) \epsilon \frac{\sinh \theta}{\theta}-(1+\tau) \cosh \theta\right] \epsilon \frac{\sinh \theta}{\theta} \\
& \nu_{3}=-\frac{\lambda}{\Omega}-\left[\tau \epsilon \frac{\sinh \theta}{\theta}-\cosh \theta\right] z \epsilon \frac{\sinh \theta}{\theta}
\end{aligned}
$$

where $\tau=(\omega+(\alpha+\beta) z) / \Omega$.

Having analyzed the quasi-Hermitian Hamiltonian $H$ given in equations (2.1), (2.2), we turn toward its pseudo-Hermitian supersymmetric extension and to the construction of supercoherent states for pseudo-Hermitian (supersymmetric) systems.

\section{Quasi-Hermitian supersymmetric extension}

Quantum-mechanical SUSY is extended to the case of general pseudo-Hermitian Hamiltonians $[2,16,18,19]$ by replacing the superalgebra of standard SUSY quantum mechanics $[20,21]$ by the pseudo-superalgebra

$$
Q^{2}=Q^{\# 2}=0, \quad\left\{Q, Q^{\#}\right\}=2 H_{s},
$$

where all operators remain $\mathbb{Z}_{2}$-graded as usual, the Hamiltonian $H_{s}$ is pseudo-Hermitian with respect to some $\mathbb{Z}_{2}$-graded operator $\eta$ : $H_{s}^{\dagger}=\eta H_{s} \eta^{-1}, Q$ is the pseudo-Hermitian (PH-SUSY) generator (supercharge) and $Q^{\#}=\eta^{-1} Q^{\dagger} \eta$ is the pseudo-adjoint of $Q$ with the same $\eta$. Mostafazadeh has explored in [16] the statistical origin of PH-SUSY quantum mechanics, showing that there exist two types of PH-SUSY realizations. The first one corresponds to exchange symmetry between a boson and phermion; in this case the metric operator is definite and the phermions are physically equivalent to the ordinary fermions. The second type, which is fundamentally different from the standard boson-fermion system, corresponds to the exchange symmetry between a boson and abnormal phermion; in this case the metric operator is indefinite.

Since our Hamiltonian $H$ given in equation (2.2) is quasi-Hermitian, the supersymmetric extension corresponding to $H$ is characterized by the boson-phermion system described by the following Hamiltonian [16]:

$$
H_{s}=H_{b}+H=\Omega\left(a^{\dagger} a+B^{\#} B\right),
$$

where $H_{b}=\Omega\left(a^{\dagger} a+\frac{1}{2}\right)$ is the bosonic contribution and $H$ is the phermionic one, given in equation (2.12); $\Omega$ is real and positive given in equation (2.8), $a^{\dagger}$ and $a$ are the standard bosonic creation and annihilation operators $\left(\left[a, a^{\dagger}\right]=\mathbf{1}\right)$, and $B^{\#}$ and $B$ are the phermionic creation and annihilation operators defined by the algebra given in equation (2.11). The bosonic operators $a$ and $a^{\dagger}$ are supposed [16] to commute with any phermionic operator constructed out of $B, B^{\#}$ and $\eta$ :

$$
\begin{aligned}
& {[a, B]=\left[a, B^{\#}\right]=[a, \eta]=0,} \\
& {\left[a^{\dagger}, B\right]=\left[a^{\dagger}, B^{\#}\right]=\left[a^{\dagger}, \eta\right]=0 .}
\end{aligned}
$$

From the third relation in (3.3) in which $a^{\dagger}$ commute with $\eta$, we have:

$$
a^{\#}=\eta^{-1} a^{\dagger} \eta=\eta^{-1} \eta a^{\dagger}=a^{\dagger} .
$$


Hence, for the bosonic operators $a^{\dagger}$ and $a$ the pseudo-Hermitian conjugation operation (\#) coincide with the conjugation operation $\left(^{\dagger}\right)$.

The equivalent Hermitian supersymmetric Hamiltonian is given by

$$
h_{s}=\rho H_{s} \rho^{-1}=\Omega\left(a^{\dagger} a+b^{\dagger} b\right)
$$

The operator $h_{s}$ is in the form of boson-fermion oscillator Hamiltonian. The supercharges $Q$ and $Q^{\#}$ associated to $H_{s}$, equation (3.2), and satisfying the equation (3.1) are given by

$$
Q=\sqrt{2 \Omega} a^{\dagger} B, \quad Q^{\#}=\sqrt{2 \Omega} a B^{\#} .
$$

These $Q$ and $Q^{\#}$ commute with the Hamiltonian (3.2),

$$
\left[Q, H_{s}\right]=0=\left[Q^{\#}, H_{s}\right]
$$

Since $H_{s}$ is quasi-Hermitian with discrete spectrum, we can introduce the complete bi-orthonormal eigenbasis $\left\{\left|\psi_{(n, \epsilon)}\right\rangle,\left|\phi_{(n, \epsilon)}\right\rangle\right\}, n=0,1,2,3 \ldots$, and $\epsilon=0,1$, associated to $H_{s}$, which satisfy

$$
\begin{aligned}
& \left\langle\phi_{(n, \epsilon)} \mid \psi_{(m, \nu)}\right\rangle=\delta_{n m} \delta_{\epsilon \nu}, \\
& \sum_{n} \sum_{\epsilon=0}^{1}\left|\phi_{(n, \epsilon)}\right\rangle\left\langle\psi_{(n, \epsilon)}\left|=\sum_{n} \sum_{\epsilon=0}^{1}\right| \psi_{(n, \epsilon)}\right\rangle\left\langle\phi_{(n, \epsilon)}\right|=\mathbf{1} .
\end{aligned}
$$

Using $h_{s}=\rho H_{s} \rho^{-1}$ we can easily establish the relations of the states $\left|\psi_{(n, \epsilon)}\right\rangle,\left|\phi_{(n, \epsilon)}\right\rangle$ to the eigenstates $|n, \epsilon\rangle$ of $h_{s}$,

$$
h_{s}|n, \epsilon\rangle=E_{n}|n, \epsilon\rangle,
$$

as follows:

$$
\left|\psi_{(n, \epsilon)}\right\rangle=\rho^{-1}|n, \epsilon\rangle
$$

and

$$
\left|\phi_{(n, \epsilon)}\right\rangle=\rho|n, \epsilon\rangle .
$$

Let us note that the structure of the Hilbert space of PH-SUSY systems remain $\mathbb{Z}_{2}$-graded as in the usual SUSY, the boson-phermion Fock space being $\mathcal{H}=\mathcal{H}_{B} \oplus \mathcal{H}_{F}[16,19]$.

\section{Supercoherent states}

We embark now on the construction of the supercoherent states (SCS) for our quasi-Hermitian SUSY Hamiltonian $H_{s}$ given in equation (3.2). We shall follow as close as possible the scheme of SCS for SUSY Hamiltonians developed in papers [22, 23, 24] and the scheme of CS for the pseudo-Hermitian Hamiltonians in $[25,26]^{2}$, generalizing both of them to the PH-SUSY case. We give first the representation space on which our SCS will be defined. The set $\left\{\left|\psi_{(n, \epsilon)}\right\rangle,\left|\phi_{(n, \epsilon)}\right\rangle\right\}$ of the eigenstates of $H$ and $H^{\dagger}$ respectively spans the Fock space on which our SCS will be defined.

As in the case CS of phermion oscillator [25] our SCS are expected to take the form of two biovercomplete and bi-normalized families, this time in the boson-phermion Fock space $\mathcal{H}_{B} \oplus \mathcal{H}_{F}$. In this scheme we need to clarify first the action of boson and phermion ladder operators on

\footnotetext{
${ }^{2}$ For more rigorous math treatment of the model see Bagarello's paper [27].
} 
the eigenstates of the Hamiltonian $H_{s}$ and its conjugate $H_{s}^{\dagger}$. In the set of eigenstates $\left|\psi_{(n, \epsilon)}\right\rangle$ of $H_{s}$, the bosonic states correspond to $\epsilon=0$, while the phermionic ones $\left|\psi_{(n, 1)}\right\rangle$ correspond to $\epsilon=1$. The boson operators $a, a^{\dagger}$ and the phermion operators $B, B^{\#}$ act on the number states $\left|\psi_{(n, \epsilon)}\right\rangle$ as follows (to be compared with the corresponding action of boson and fermion ladder operators in the case of ordinary SUSY [23]):

$$
\begin{aligned}
& a\left|\psi_{(n, \epsilon)}\right\rangle=\sqrt{n}\left|\psi_{(n-1, \epsilon)}\right\rangle, \quad a^{\dagger}\left|\psi_{(n, \epsilon)}\right\rangle=\sqrt{n+1}\left|\psi_{(n+1, \epsilon)}\right\rangle, \\
& B\left|\psi_{(n, 0)}\right\rangle=0, \quad B\left|\psi_{(n, 1)}\right\rangle=\left|\psi_{(n, 0)}\right\rangle, \\
& B^{\#}\left|\psi_{(n, 1)}\right\rangle=0, \quad B^{\#}\left|\psi_{(n, 0)}\right\rangle=\left|\psi_{(n, 1)}\right\rangle .
\end{aligned}
$$

The operator $B$ annihilates the bosonic states $\left|\psi_{(n, 0)}\right\rangle$, and $B^{\#}$ maps these state onto the phermionic states $\left|\psi_{(n, 1)}\right\rangle$. The boson-phermion ground state is $\left|\psi_{(0,0)}\right\rangle$ which satisfies the equations

$$
\begin{aligned}
& a\left|\psi_{(0,0)}\right\rangle=B\left|\psi_{(0,0)}\right\rangle=0, \\
& Q\left|\psi_{(0,0)}\right\rangle=Q^{\#}\left|\psi_{(0,0)}\right\rangle=0 .
\end{aligned}
$$

The operators $Q$ and $Q^{\#}$ act on the states $\left|\psi_{(n, 1)}\right\rangle$ and $\left|\psi_{(n, 0)}\right\rangle$ as raising and lowering operators:

$$
Q\left|\psi_{(n, 1)}\right\rangle=\sqrt{\Omega(n+1)}\left|\psi_{(n+1,0)}\right\rangle, \quad Q^{\#}\left|\psi_{(n, 0)}\right\rangle=\sqrt{\Omega n}\left|\psi_{(n-1,1)}\right\rangle
$$

The operator $Q$ maps phermionic states onto bosonic ones, and $Q^{\#}$ maps bosonic states onto phermionic ones.

After having introduced all the ingredients, we construct the SCS $|\alpha, \xi\rangle$ associated to the Hamiltonian (3.2) as the orbit of the ground state (4.1) under the action of a pseudo-unitary displacement operators $D(\alpha, \xi)$ which realize a pseudo-Hermitian generalization of the representation of the Heisenberg-Weyl super algebra, generated by the boson and phermion operators $a, a^{\dagger}, B, B^{\#}$ and the identity $\mathbf{1}$ :

$$
\begin{aligned}
& |\alpha, \xi\rangle=D(\alpha, \xi)\left|\psi_{(0,0)}\right\rangle, \\
& D(\alpha, \xi)=e^{\left(\alpha a^{\dagger}-\alpha^{*} a+i \beta \mathbf{1}+B^{\#} \xi-\xi^{*} B\right)},
\end{aligned}
$$

where $\alpha$ is c-number, $\beta$ is real number, and $\xi$ is complex Grassmann number [28, 29, 30, 31]. Let us recall that $\xi$ is nilpotent and anticommutes with its conjugate,

$$
\xi^{2}=0, \quad \xi^{* 2}=0, \quad \xi \xi^{*}+\xi^{*} \xi=0 .
$$

The integrations over $\xi$ and $\xi^{*}$ are performed according to the Berezin rules,

$$
\int d \xi^{*} d \xi \xi \xi^{*}=1, \quad \int d \xi^{*} d \xi \xi=\int d \xi^{*} d \xi \xi^{*}=\int d \xi^{*} d \xi 1=0 .
$$

As in the fermion case [30] $\xi$ 's commute with ordinary complex numbers and boson operators, and anticommute with phermion operators $B$ and $B^{\#}$,

$$
\{\xi, B\}=0, \quad\left\{\xi^{*}, B\right\}=0, \quad\left\{\xi, B^{\#}\right\}=0, \quad\left\{\xi^{*}, B^{\#}\right\}=0 .
$$

The pseudo-Hermitian conjugation reverses the order of all fermionic quantities, both the operators and the Grassmann numbers:

$$
\left(B^{\#} \xi+\xi^{*} B\right)^{\#}=\xi^{*} B+B^{\#} \xi .
$$


By using the Baker-Campbell-Hausdorff formulas [32] the displacement operators $D(\alpha, \xi)$ is written (up to a constant phase factor) in the form:

$$
D(\alpha, \xi)=e^{\left(-\frac{1}{2} \xi^{*} \xi-\frac{|\alpha|^{2}}{2}\right)} e^{\alpha a^{\dagger}} e^{-\xi B^{\#}} e^{-\alpha^{*} a} e^{-\xi^{*} B} .
$$

The pseudo-Hermitian adjoint $D^{\#}(\alpha, \xi)$ is given by

$$
D^{\#}(\alpha, \xi)=e^{-\left(\alpha a^{\dagger}-\alpha^{*} a+B^{\#} \xi-\xi^{*} B\right)} .
$$

In the last expression of $D^{\#}(\alpha, \xi)$, we have taken into account that for all the bosonic operators, the pseudo-Hermitian conjugation operation $\left(^{\#}\right)$ coincides with the conjugation operation $\left({ }^{\dagger}\right)$, which is expressed in the equation (3.3) as consequence of the fact that the bosonic operators $a^{\dagger}$ and $a$ commutes with $\eta[16]$.

The displacement operator $D$ is pseudo-unitary: $D^{\#} D=\mathbf{1}=D D^{\#}$. The substitution of the expression (4.7) of $D(\alpha, \xi)$ in the equation (4.2) yields the following expression of SCS $|\alpha, \xi\rangle$,

$$
|\alpha, \xi\rangle=e^{-\frac{1}{2} \xi^{*} \xi}(|\alpha, 0\rangle-\xi|\alpha, 1\rangle) .
$$

Here $|\alpha, 0\rangle$ are the standard boson CS (Glauber CS [33]) given explicitly by

$$
|\alpha, 0\rangle=e^{-\frac{|\alpha|^{2}}{2}} \sum_{n=0}^{\infty} \frac{\alpha^{n}}{\sqrt{n !}}\left|\psi_{(n, 0)}\right\rangle,
$$

$\left|\psi_{(n, 0)}\right\rangle \equiv|n\rangle$ representing the Fock space for the standard bosonic harmonic oscillator. The states $|\alpha, 1\rangle$ are the phermionic states given explicitly by

$$
|\alpha, 1\rangle=e^{-\frac{|\alpha|^{2}}{2}} \sum_{n=0}^{\infty} \frac{\alpha^{n}}{\sqrt{n !}}\left|\psi_{(n, 1)}\right\rangle .
$$

In the limit $\xi=0$, the expression (4.8) of $|\alpha, \xi\rangle$ recovers the standard boson CS (4.9), i.e. the PH-SUSY superalgebra is reduced to the standard boson algebra.

The Hermitian adjoint of $|\alpha, \xi\rangle$ is

$$
\langle\alpha, \xi|=e^{-\frac{1}{2} \xi^{*} \xi}\left(\langle\alpha, 0|+\xi^{*}\langle\alpha, 1|\right)
$$

and the inner product $\langle\alpha, \xi \mid \alpha, \xi\rangle \neq 1$, which is due to the nonorthogonality of the states $\left|\psi_{(n, \epsilon)}\right\rangle$, the latter property being a consequence of the fact that $B^{\#} \neq B^{\dagger}$.

Now we have to examine for (over)completeness the set of $|\alpha, \xi\rangle$. One can check (using the rules (4.3)-(4.6)) that the resolution of identity is not satisfied, because the states $\left|\psi_{(n, \epsilon)}\right\rangle$ do not form an orthogonal basis, which is a consequence of the non-Hermiticity of the Hamiltonian (3.2):

$$
\int|\alpha, \xi\rangle\langle\alpha, \xi| d \mu(\alpha) d \xi^{*} d \xi \neq \mathbf{1}, \quad d \mu(\alpha)=d \alpha^{*} d \alpha / \pi
$$

The useful way to solve this problem of the (over)completeness, is to use the main idea introduced previously for the case of pseudo-Hermitian CS in [25, 26], which consists of introduction of a complementary pair of lader operators, such that the system of two complementary sets of CS forms the so-called bi-orthonormal and bi-overcomplete system. In this aim we introduce the second dual ladder operator $\tilde{B}$ which is associated to $H^{\dagger}$. The $B$ and $\tilde{B}$ form a complementary pair of lader lowering operators. $\tilde{B}$ is related to the annihilation operator $b$ of $h$ via the similarity transformation:

$$
\tilde{B}=\rho b \rho^{-1} .
$$


Its action on the eigenstates of $H^{\dagger}$ is

$$
\tilde{B}\left|\phi_{(n, 0)}\right\rangle=0, \quad \tilde{B}\left|\phi_{(n, 1)}\right\rangle=\left|\phi_{(n, 0)}\right\rangle .
$$

The operator $\tilde{B}$ is nilpotent. The operator $B^{\dagger}=\rho b^{\dagger} \rho^{-1}$ is the creation operator for $H^{\dagger}$. In this way one obtains a second pair of phermionic lowering and raising operators $\tilde{B}$ and $B^{\dagger}$,

$$
\begin{aligned}
& \tilde{B} B^{\dagger}+B^{\dagger} \tilde{B}=1, \quad \tilde{B}^{2}=B^{\dagger 2}=0, \\
& B^{\dagger}\left|\phi_{(n, 1)}\right\rangle=0, \quad B^{\dagger}\left|\phi_{(n, 0)}\right\rangle=\left|\phi_{(n, 1)}\right\rangle .
\end{aligned}
$$

In view of the phermionic algebra (4.10) we introduce new displacement super operators $\widetilde{D}(\alpha, \xi)$,

$$
\widetilde{D}(\alpha, \xi)=e^{\left(\alpha a^{\dagger}-\alpha^{*} a+B^{\dagger} \xi-\xi^{*} \tilde{B}\right)} .
$$

We build up now the second family of SCS $\widetilde{|\xi, \alpha\rangle}$ according to the above described scheme (see equations (4.2), (4.8)),

$$
\widetilde{|\alpha, \xi\rangle}=\widetilde{D}(\alpha, \xi)\left|\phi_{(n, 0)}\right\rangle=e^{-\frac{1}{2} \xi^{*} \xi}(\widetilde{|\alpha, 0\rangle}-\xi \widetilde{|\alpha, 1\rangle}) \text {, }
$$

where $\left|\phi_{(n, 0)}\right\rangle$ is the ground state of $H_{s}^{\dagger}, \widetilde{|\alpha, 0\rangle}$ and $\widetilde{|\alpha, 1\rangle}$ being given explicitly by,

$$
\widetilde{|\alpha, 0\rangle}=e^{-\frac{|\alpha|^{2}}{2}} \sum_{n=0}^{\infty} \frac{\alpha^{n}}{\sqrt{n !}}\left|\phi_{(n, 0)}\right\rangle, \quad \widetilde{|\alpha, 1\rangle}=e^{-\frac{|\alpha|^{2}}{2}} \sum_{n=0}^{\infty} \frac{\alpha^{n}}{\sqrt{n !}}\left|\phi_{(n, 1)}\right\rangle .
$$

Since the eigenstates $\left|\phi_{(n, \epsilon)}\right\rangle$ of $H_{s}^{\dagger}$ are not orthogonal, the scalar product $\left.\widetilde{\langle\alpha, \xi|} \widetilde{\alpha, \xi}\right\rangle$, like the previous one $\langle\alpha, \xi \mid \alpha, \xi\rangle$, is different from 1 . The two subsets of states $\{|\alpha, \xi\rangle\}$ and $\{\widetilde{|\alpha, \xi\rangle}\}$ are bi-normalized instead:

$$
\widetilde{\langle\alpha, \xi}|\alpha, \xi\rangle=\langle\alpha, \xi \widetilde{\alpha, \xi\rangle}=1 .
$$

By means of the two type of states $|\alpha, \xi\rangle$ and $\widetilde{|\alpha, \xi\rangle}$ the resolution of the identity is realized in the following way,

$$
\int|\alpha, \xi\rangle \widetilde{\langle\alpha, \xi|} d \mu(\alpha) d \xi^{*} d \xi=\int \widetilde{|\alpha, \xi\rangle}\langle\alpha, \xi| d \mu(\alpha) d \xi^{*} d \xi=\mathbf{1}
$$

The equation (4.12) can be easily verified using the formulas of $|\alpha, \xi\rangle$ and $\widetilde{|\alpha, \xi\rangle}$ (equations (4.8) and (4.11)) and the rules of integration (4.4). Thus the system of states $\{|\alpha, \xi\rangle, \widetilde{|\alpha, \xi\rangle}\}$ is biovercomplete in boson-phermion Fock space. It is this system that we call boson-phermion SCS, or more shortly pseudo-Hermitian SCS.

We would like to emphasize that these SCS satisfy also the first definition of usual coherent states (CS) given by Glauber [33] as eigenstates of boson and phermion annihilation operators:

$$
\begin{array}{ll}
a|\alpha, \xi\rangle=\alpha|\alpha, \xi\rangle, & B|\alpha, \xi\rangle=\xi|\alpha, \xi\rangle, \\
a \widetilde{|\alpha, \xi\rangle}=\alpha \widetilde{\alpha, \xi\rangle,}, & \tilde{B} \widetilde{\alpha, \xi\rangle}=\xi \widetilde{\alpha, \xi\rangle} .
\end{array}
$$

Finally, in the limit of $\eta=1$ (that is $B^{\#} \equiv B^{\dagger}$ ), our SCS recover the standard SCS for SUSY boson-fermion oscillator [23, 24]. In the double limits of $\eta=1$ and $\xi=0$, these SCS coincide with of the standard Glauber's CS [33]. 


\section{Concluding remarks}

In this paper, we have achieved some extensions in the framework of the pseudo-Hermitian quantum mechanics. We have extended the study of the Hermitian su(2) Hamiltonians to the case of non-Hermitian $\mathrm{su}(2)$ Hamiltonians with real spectrum. For such pseudo-Hermitian Hamiltonian system we established the metrics which allows the transition to the corresponding Hermitian Hamiltonian. The constructed metric operator depends on one real parameter as in the case of the $\mathrm{su}(1,1)$ approach, this time however the real parameter is not restricted by any inequality.

We have also extended the supercoherent states (SCS) approach to pseudo-Hermitian supersymmetric (PH-SUSY) system characterized by the boson-phermion oscillator [16, 19]. The supersymmetric displacement operator method and the ladder operator method for construction of ordinary SCS $[23,24]$ are both extended to the case PH-SUSY systems. For the bosonphermion systems there are two complimentary pairs of ladder operators which have to be used to construct bi-orthonormal Fock states and coherent states. As a result the set of constructed SCS for the boson-phermion system consists of a pair of two (dual) subsets of states which are bi-normal and form a bi-overcomplete system in the corresponding Hilbert space. The states of each subset are eigenvectors of the boson annihilation operator and of the corresponding phermionic lowering operator. In the limit of Hermitian SUSY system our states recover the known SCS [23, 24].

The PH-SUSY and pseudo-SCS scheme, developed in this paper for the boson-phermion systems, can be naturally extended and applied to study other (more general) PH-SUSY systems, such as system of pseudo-bosons [26] and fermions and system of pseudo-bosons and phermions.

\section{References}

[1] Bender C.M., Boettcher S., Real spectra in non-Hermitian Hamiltonians having PT symmetry, Phys. Rev. Lett. 80 (1998), 5243-5246, math-ph/9712001.

[2] Mostafazadeh A., Pseudo-Hermiticity versus PT symmetry: The necessary condition for the reality of the spectrum of a non-Hermitian Hamiltonian, J. Math. Phys. 43 (2002), 205-214, math-ph/0107001.

[3] Swanson M.S., Transition elements for a non-Hermitian quadratic Hamiltonian, J. Math. Phys. 45 (2004), 585-601.

[4] Geyer H.B., Scholtz F.G., Snyman I., Quasi-Hermiticity and the role of a metric in some boson Hamiltonians, Czechoslovak J. Phys. 54 (2004), 1069-1073.

[5] Jones H.F., On pseudo-Hermitian Hamiltonians and their Hermitian counterparts, J. Phys. A: Math. Gen. 38 (2005), 1741-1746, quant-ph/0411171.

[6] Bagchi B., Quesne C., Roychoudhury R., Pseudo-Hermiticity and some consequences of a generalized quantum condition, J. Phys. A: Math. Gen. 38 (2005), L647-L652, quant-ph/0508073.

[7] Musumbu D.P., Geyer H.B., Heiss W.D., Choice of a metric for the non-Hermitian oscillator, J. Phys. A: Math. Theor. 40 (2007), F75-F80, quant-ph/0611150.

[8] Quesne C., A non-Hermitian oscillator Hamiltonian and su(1,1): a way towards generalizations, J. Phys. A: Math. Theor. 40 (2007), F745-F751, arXiv:0705.2868.

[9] Quesne C., Quasi-Hermitian supersymmetric extensions of a non-Hermitian oscillator Hamiltonian and of its generalizations, J. Phys. A: Math. Theor. 41 (2008), 244022, 10 pages, arXiv:0710.2453.

[10] Bagchi B., Quesne C., Non-Hermitian Hamiltonians with real and complex eigenvalues in a Lie-algebraic framework, Phys. Lett. A 300 (2002), 18-26, math-ph/0205002.

[11] Assis P.E.G., Fring A., Non-Hermitian Hamiltonians of Lie algebraic type, J. Phys. A: Math. Theor. 42 (2009), 015203, 23 pages, arXiv:0804.4677.

[12] Allen L., Eberly J.H., Optical resonance and two-level atoms, Dover Publications, Inc., New York, 1987.

[13] Wódkiewicz K., Eberly J.H., Coherent states, squeezed fluctuations, and the SU(2) and SU(1,1) groups in quantum-optics applications, J. Opt. Soc. Am. B 2 (1985), 458-466. 
[14] Arecchi F.T., Courtens E., Gilmore R., Thomas H., Atomic coherent states in quantum optics, Phys. Rev. A 6 (1972), 2211-2237.

[15] Zhang W.M., Feng D.H., Gilmore R., Coherent states: theory and some applications, Rev. Modern Phys. 62 (1990), 867-927.

[16] Mostafazadeh A., Statistical origin of pseudo-Hermitian supersymmetry and pseudo-Hermitian fermions, J. Phys. A: Math. Gen. 37 (2004), 10193-10207, quant-ph/0404025.

[17] Cherbal O., Drir M., Maamache M., Trifonov D.A., Invariants and coherent states for a nonstationary fermionic forced oscillator, Phys. Lett. A 374 (2010), 535-538, arXiv:0912.4820.

[18] Mostafazadeh A., Pseudo-supersymmetric quantum mechanics and isospectral pseudo-Hermitian Hamiltonians, Nuclear Phys. B 640 (2002), 419-434, math-ph/0203041.

[19] Mostafazadeh A., Pseudo-Hermitian supersymmetry: a brief review, Czechoslovak J. Phys. 54 (2004), 13711374 .

[20] Gendenshtein L.E., Krive I.V., Supersymmetry in quantum mechanics, Soviet Phys. Uspekhi 28 (1985), 645-666.

[21] Cooper F., Khare A., Sukhatme U., Supersymmetry in quantum mechanics, World Scientific Publishing Co., Inc., River Edge, NJ, 2001.

[22] Aragone C., Zypman F., Supercoherent states, J. Phys. A: Math. Gen. 19 (1986), 2267-2279.

[23] Fatyga B.W., Kostelecký V.A., Nieto M.M., Truax D.R., Supercoherent states, Phys. Rev. D 43 (1991), 1403-1412.

[24] Berube-Lauziere Y., Hussin V., Comments of the definitions of coherent states for the SUSY harmonic oscillator, J. Phys. A: Math. Gen. 26 (1993), 6271-6275.

[25] Cherbal O., Drir M., Maamache M., Trifonov D.A., Fermionic coherent states for pseudo-Hermitian two-level systems, J. Phys. A: Math. Gen. 40 (2007), 1835-1844, quant-ph/0608177.

[26] Trifonov D.A., Pseudo-boson coherent and Fock states, in Proceedings of the 9th International Workshop on "Complex Structures, Integrability and Vector Fields" (August 25-29, 2008, Sofia), Editors K. Sekigawa, V. Gerdjikov and S. Dimiev, World Scientific Publishing Co., 2009, 241-250, arXiv:0902.3744.

[27] Bagarello F., Pseudobosons, Riesz bases, and coherent states, J. Math. Phys. 51 (2010), 023531, 10 pages, arXiv:1001.1136.

[28] Berezin F.A., The method of second quantization, Academic Press, New York, 1966.

[29] Berezin F.A., Marinov M.S., Particle spin dynamics as the Grassmann variant of classical mechanics, Ann. Physics 104 (1977), 336-362.

[30] Cahill K.E., Glauber R.J., Density operators for fermions, Phys. Rev. A 59 (1999), 1538-1555, physics/9808029.

[31] Junker G., Klauder J.R., Coherent-state quantization of constrained fermion systems, Eur. Phys. J. C 4 (1998), 173-183, quant-ph/9708027.

[32] Gilmore R., Baker-Campbell-Hausdorff formulas, J. Math. Phys. 15 (1974), 2090-2092.

[33] Glauber R.J., Coherent and incoherent states of the radiation field, Phys. Rev. 131 (1963), 2766-2788. 